\title{
ANALYSIS OF ECONOMIC PROCESSES AND SYSTEMS BASING ON THE USE OF ECONOMIC AND MATHEMATICAL METHODS AND INSTRUMENTS
}

\section{EXPERT REVIEW: METHOD OF INTUITIVELY AGREED CHOICE}

\section{G. Khubaev ${ }^{1}$}

\section{Abstract}

Suggested here is a method of intuitively agreed collective choice in the process of expert review of *the best project, *best expert, *best appearance design, *best problem solution. That being the case, the experts: are unaware of who participates in the survey; are unaware of who made what decision; are unaware of who and how rationalized their decisions, and cannot know true goals of expert review survey organizers. At the same time, the experts can obtain statistical characteristics of an attribute in question during simulation modeling.

Keywords: expert review, agreed choice, solutions, Kemeny distance and median, simulation modeling, statistical characteristics.

doi: http:doi.org/10.15350/UK_3/5/3

Thesis Statement supposes that administrative structures of various socio-economic systems - government of a country, authority of an administrativeterritorial entity, board of directors of a large company, etc., - have ordered from renowned specialists development of a project aimed at solution of a complex problem, topical for large social groups. For the sake of order implementation, $\boldsymbol{n}$ groups of specialists have offered their projects (techniques, methods, algorithms) for solution of a particular issue (economical, technical, military, environmental, etc.).

[Examples of such, permanently requiring solution of complex socio-economic issues: Methods (promptly and with the minimum labor, material, energy and financial resources) that can *improve public welfare; *budget income, *ensure higher growth of GDP, tax revenue; or *improve competitive market positions of one of the (country's, world) largest banks, organizations, etc.]

The question is which of $\boldsymbol{n}$ ways of solving a topical issue presented is the most appropriate according to the established criterion under specific implementation conditions? And how can it be determined correctly, objectively, reasonably? The projects were prepared by different groups of specialists, with different professional competences, different creative abilities, different ambitions

\footnotetext{
${ }^{1}$ Georgy Khubaev, dr of Economics, professor, Rostov State University of Economics, Russia.
} 
and different extent of interest in results of objective solution of that complex issue. Besides, each of the $\boldsymbol{n}$ groups is sure that it is their project that is the best, the most effective.

Now the top level system - government, ministry, board of directors, etc. has to choose the best solution according to the established criteria out of $\boldsymbol{n}$ solutions for the topical issue. But it is no easy task.

What challenges are faced during implementation of the procedure of choice?

First of all, when choosing any of $\boldsymbol{n}$ competing issue solutions there are always people among the developers of rejected projects and their supporters who are not satisfied with the results of expert choice, per the following saying: Says who?!, i.e. challenge one is to select expert members for comparative assessment of competing projects per criterion established by the project originator. Expert review of projects must be performed by people who are competent, talented, responsible and interested in the appropriate solution of an issue in question. But such people still need to be found!

Second of all, currently there is no clear understanding as to what the assessment procedure for appropriateness, objectivity, efficiency of complex task solution should be. Which is why challenge two is the search for procedure of generation of collective choice during expert review. And management bodies of all levels have to face such challenges on a regular basis. At the same time, as a rule, the error in the choice is associated with very significant losses of resources invested in the implementation of the project: with potential worsening of public welfare, company bankruptcy, unemployment increase, waste of resources, etc.

Let us say, that the task of comparative assessment and the choice of a solution for complex issue is a set of individual tasks.

Task 1. - Forming the composition of experts reviewing issue solutions.

Task 2. - Analysis and ranking factors affecting implementation processes and effectiveness of each of projects (ways) for solving issue in question presented.

Task 3. - Quantitative assessment for each project (issue solution): *labor, material, energy and financial resources spent for realization and implementation of the projects; *time from the investment of funds to receipt of benefit (time lagging, delay); *economic efficiency of the projects.

Task 4. Analysis of results of comparative quantitative assessment of the attributes characterizing effectiveness of issue solutions suggested (results of Task 2 and Task 3 solution) and realization of the procedure of the choice of the best solution.

The article offers a method of forming intuitively agreed collective choice during comparative expert review of effectiveness of solutions for a complex issue suggested by different groups of specialists. Procedure of choosing the best solution suggests implementation of two following stages: the first stage includes selection of experts with professional competences and creative abilities, and the second stage includes generation of agreed collective choice during expert review. 


\section{Preliminary remarks.}

1) With the development of science and technology, even a very responsible and capable person can acquire less and less topical information. So, nowadays due to continuous acceleration of scientific and technological progress, reduction of the period of knowledge doubling, snowballing growth in the number of publications in most areas of science and technology, few people succeed in retaining the advanced positions for a long time, even in one narrow area of knowledge. According to the literature, in order to deal with new theory and apply it in practice, even a well-trained professional often requires years of hard work. Respectively, intensity of the use of expert method in foreseeable future will only increase.

2) People are known to differ significantly in both, their professional knowledge level, and abilities they have been gifted by nature. Thus, out of hundred million chess players only 3-5 persons can play 20 games "blindly". It is, therefore, obvious that a person who "sees" 4 steps ahead (or is capable of visualizing 4 steps of task solution algorithm), will not be able to convince a person who is capable of seeing only 2 ahead of the correctness, good reason behind their decision (when it, for instance, concerns choice of economy development paths. In a game of chess everything is way simpler: correctness can easily be proved by a real game, while error in calculations will not adversely impact lives of other people). Also, depending on the natural abilities of an individual, time spent to learn new material, to acquire new knowledge will differ as well. Experiments (see [1]), therefore, once again prove a very significant influence of individual abilities on speed of realizing opportunities for practical use of knowledge obtained and actual realization of such opportunities. It turned out that under the same ambient conditions and the same initial level of knowledge of theoretical theses, time sufficient for practical use of newly learned material, has a significant variance, significant dextrality and can, often times, be approximated by alphadistribution (see [2]). Later on, author's students confirmed that with the same level of initial training and under the same experiment conditions dextrality of time consumption distribution for performance of works increases along with the increase of complexity of works.

That is why the thesis is deemed quite real and sound that every thousand of physicists, medical specialists, economy specialists, and representatives of other trades would hardly yield a couple of dozens of people, who a) are at the leading edge of a particular science and b) who are potentially, technically capable of understanding and appropriately assessing a new theory, results, ideas or suggestions of other member of this small groups of professionals (it is worthwhile highlighting that we only speak about those people, who possess abilities and required expertise. But this still does not mean that such a professional would, firstly, want to figure out the ideas of competing colleague, and, secondly, having figured them out, be unbiased and honest in his/her assessment).

3) Identifying an expert possessing maximum expertise in a particular discipline is a no easy task. This can easily be proven. Figure 1 shows Venn diagram depicting knowledge level that individuals $\boldsymbol{A}-\boldsymbol{E}$ communicating with one another have. It is quite obvious that individual $\boldsymbol{A}$ would hardly have doubts that individual $\boldsymbol{C}$, with whom $\boldsymbol{A}$ has much in common, is cleverer than individual $\boldsymbol{B}$. $\boldsymbol{B}$, 
in his/her turn, would (due to the same reasons) believe that $\boldsymbol{D}$ is cleverer than $\boldsymbol{A}$. But they would all be convinced that $\boldsymbol{E}$ is a quite a narrow-minded person despite that fact that in reality the latter has a way greater knowledge as compared to $\boldsymbol{A}, \boldsymbol{B}, \boldsymbol{C}$ and $\boldsymbol{D}$ altogether.

Under such conditions, individual $\boldsymbol{E}$ cannot prove higher effectiveness (performance, usefulness) of his/her suggestion to individuals $\boldsymbol{A}$ or $\boldsymbol{B}$, if he/she uses knowledge that is not overlapped with those of $\boldsymbol{A}$ and $\boldsymbol{B}$ as a proof. But unlike the case with innate abilities, lack of understanding of $\boldsymbol{E}$ 's viewpoint is due to difference of scope of knowledge of separate individuals.

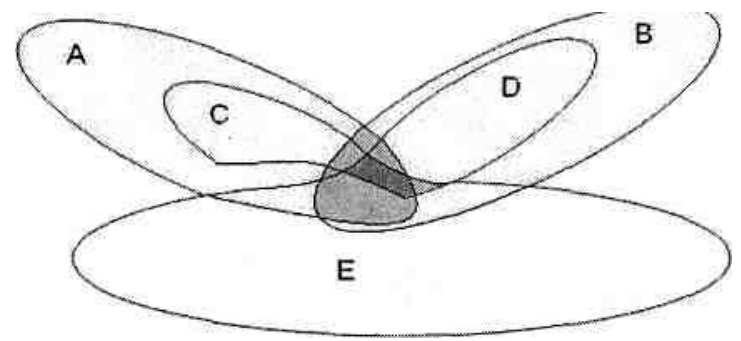

Figure 1. Scope of knowledge individuals $\boldsymbol{A}-\boldsymbol{E}$ possess.

\section{SUGGESTED METHODS FOR SOLVING HE FOUR AFOREMENTIONED TASKS}

1. Forming the Composition of Expert Team (Task 1). Tasks related to selection of experts for comparative assessment of effectiveness and reasoning of solutions to issues suggested are faced on a quite regular basis. Which is why it is deemed reasonable to create (centralized) a uniform database (DB) that contains information about all potential participants of expert review of the kind. Information contained in the DB may include publicly available data on the publications in a particular field, number of times expert's works have been quoted, successful experience of participation in expert reviews, etc. What is more, algorithms described in $[3,4]$ can be used for generation of such database.

Let us assume that a required subset of potential experts has been generated. Below are further activities:

1.1. Subset of expert review participants (reviewers of competing projects) generated is divided into two groups: subgroup $\mathbf{G 1}$ develops quantitative assessments for quality attributes for each project - it solves Tasks 2 and 3, subgroup $\mathbf{G} \mathbf{2}$ - analyzes the results of expert review and chooses the best project, i.e. it solves Task 4.

1.2. Subgroup $\mathbf{G 1}$ is extended to perform a collective qualitative expert review of the projects through adding $\boldsymbol{n}$ specialist groups suggesting their projects (solutions to issues in question).

Below the advantages of including all developers of competing projects to subgroup G1 are listed. Firstly, each of the developers, as a rule, knows peculiarities of their projects and can, therefore, reasonably add up the content of factors affecting project performance, specify advantages and refute noted deficiencies of their projects, i.e. they can actively, often times, unconsciously participate in 
solving Tasks 2 and 3. Figure 3 shows graph A depicting correlation (particular coherence) between the answers of experts. Group A mainly includes experts from subgroup G1, but experts 1 and 4, also included to group A, are the developers of competing projects. Secondly, since the developers, participating in expert review, are aware of both, all justifications of the experts supporting answers that differ greatly, and the results of answer processing after each round of survey, probability of criticism of expert review results reduces significantly.

1.3. All $\boldsymbol{N}$ participants of expert review (selected from $\boldsymbol{n}, \boldsymbol{G 1}$ and $\boldsymbol{G} \mathbf{2}$ groups) are assigned identifiers from a random numbers table (or generator) and granted free Internet access.

2. Identifying and Ranking Determinant Factors (Task 2). At the first stage of the expert review, factors affecting processes of implementation and performance from each of $\boldsymbol{n}$ projects need to be identified and sorted. But how can this be done? Which of the available methods of expert review should be used?

We believe that it is justified the use of the method of PURO [5, 6] - stepby-step refinement of ranzhirovany objects (factors, characteristics, actors, indicators, etc.) based on the integration of the Delphi procedures for the prediction of the future (developed by O. Helmer, N. Dalkey, T.J. Gordon - see, e.g., [7, 8]) with assessment of distance and median between factor-object rankings offered by Kemeny J.G. $[9,10]$.

2.1. Description of PURO method. If $\mathbf{m}$ experts need to rank $\boldsymbol{k}$ factors by the extent of their impact on the performance of $\mathbf{n}_{\mathbf{i}}$ project, a series of questionnaires is performed. Once the results of a questionnaire have been processed, a median, smallest and largest ranks are assigned to all $\boldsymbol{k}$ factors in questionnaires that support greatly differing responses. Each ranking is presented in the form of a canonical sorting matrix, and then measure of Kemeny proximity (distance) between all rankings is calculated. Kemeny distance $\mathbf{d}_{\mathbf{i j}}$ characterizes the extent of discrepancy between the rankings of two experts $\left(\mathbf{d}_{\max }=\mathbf{n}(\mathbf{n}-\mathbf{1})\right)$. Matrix $\mathbf{D}=\left\{\mathbf{d}_{\mathrm{ij}}\right\},(\mathbf{i}, \mathbf{j} \in \mathbf{m})$ will show all $(\mathbf{m}-\mathbf{1}) \mathbf{m} / \mathbf{2}$ of distances between the rankings. $\mathbf{D}$ symmetrical positive matrix with zero diagonal elements. Sum of items of $\mathbf{i}$ row of matrix $\mathbf{D}$ corresponds to the value of discrepancy between expert $\mathbf{i}$ and all other experts. Comparing sums of all elements of matrices $\mathbf{D}$ obtained after each questionnaire, speed of experts' opinions repeatability can be assessed, the most (the least) accorded rankings can be identified and consistent group of experts can be singled out (by selecting different thresholds of proximity $\mathbf{d}_{\mathrm{ij}} \leq \boldsymbol{\varepsilon}_{\mathrm{d}}$ ). If experts' opinions match completely relative to factor rankings, $\mathbf{d}_{\mathbf{i j}} \mathbf{=} \mathbf{0}$, if they contradict $-\mathbf{d}^{\mathbf{0}_{\mathbf{i j}}=\mathbf{1} \text {. }}$

2.2. Classification (Grouping) Expert Questionnaire Participants. Let us assume that the procedure of increment sorting of multiple factors has been completed successfully. Matrix of Kemeny distance between all expert rankings has been generated. The question is how matched response groups from experts can be identified correctly?

Let us take a look at the procedure of search for matched groups of experts' responses (expert classes) and selection of consistent sorting of responses for such identified group.

Assume that the stepwise sorting of the set of eight factors $(m=8)$ by nine experts $(n=9)$ resulted in Kemeny ranking distances $\left\{\mathbf{d}_{\mathrm{ij}}\right\}$ shown in Table 1. 
Table 1

Kemeny distances between expert (response) rankings

\begin{tabular}{|c|c|c|c|c|c|c|c|c|c|}
\hline$\left\{d_{i j}\right\}$. & E1 & E2 & E3 & E4 & E5 & E6 & E7 & E8 & E9 \\
\hline E1 & 0 & 2 & 28 & 4 & 4 & 18 & 4 & 2 & 2 \\
\hline E2 & 2 & 0 & 42 & 4 & 2 & 35 & 2 & 4 & 4 \\
\hline E3 & 28 & 42 & 0 & 24 & 36 & 14 & 42 & 36 & 24 \\
\hline E4 & 4 & 4 & 24 & 0 & 4 & 48 & 2 & 2 & 2 \\
\hline E5 & 4 & 2 & 36 & 4 & 0 & 48 & 2 & 4 & 2 \\
\hline E6 & 18 & 35 & 14 & 48 & 48 & 0 & 28 & 42 & 32 \\
\hline E7 & 4 & 2 & 42 & 2 & 2 & 28 & 0 & 2 & 4 \\
\hline E8 & 2 & 4 & 36 & 2 & 4 & 42 & 2 & 0 & 4 \\
\hline E9 & 2 & 4 & 24 & 2 & 2 & 32 & 4 & 4 & 0 \\
\hline
\end{tabular}

To identify matched groups of experts the following steps are taken:

Step 1. Convert elements of distance matrix to relative units using expression $\mathbf{d} \mathbf{i j}_{\mathbf{i j}}=\mathbf{d}_{\mathrm{ij}} / \mathbf{d}_{\text {max }}$. Maximum distance between factor rankings set forth in Table 1 equals to $\mathbf{d}_{\max }=m(m-1)=8^{*} 7=56$. As a result, we get Table 2 with Kemeny distances in relative units $\left\{\mathbf{d}^{\mathbf{0}}{ }_{\mathbf{i j}}\right\}$.

Table 2

Kemeny distances between rankings in relative units

\begin{tabular}{|c|c|c|c|c|c|c|c|c|c|}
\hline$\left\{\mathrm{d}^{0}{ }_{\mathrm{ij}}\right\}$. & E1 & E2 & E3 & E4 & E5 & E6 & E7 & E8 & E9 \\
\hline E1 & 0 & 0.04 & 0.5 & 0.07 & 0.07 & 0.3 & 0.07 & 0.04 & 0.04 \\
\hline E2 & 0.04 & 0 & 0.75 & 0.07 & 0.04 & 0.6 & 0.04 & 0.07 & 0.07 \\
\hline E3 & 0.5 & 0.75 & 0 & 0.4 & 0.6 & 0.25 & 0.75 & 0.6 & 0.4 \\
\hline E4 & 0.07 & 0.07 & 0.4 & 0 & 0.07 & 0.86 & 0.04 & 0.04 & 0.04 \\
\hline E5 & 0.07 & 0.04 & 0.6 & 0.07 & 0 & 0.86 & 0.04 & 0.07 & 0.04 \\
\hline E6 & 0.3 & 0.6 & 0.25 & 0.86 & 0.86 & 0 & 0.5 & 0.75 & 0.57 \\
\hline E7 & 0.07 & 0.04 & 0.75 & 0.04 & 0.04 & 0.5 & 0 & 0.04 & 0.07 \\
\hline E8 & 0.04 & 0.07 & 0.6 & 0.04 & 0.07 & 0.75 & 0.04 & 0 & 0.07 \\
\hline E9 & 0.04 & 0.07 & 0.4 & 0.04 & 0.04 & 0.57 & 0.07 & 0.07 & 0 \\
\hline
\end{tabular}

Step 2. Based on real extent of experts' response correspondence, select

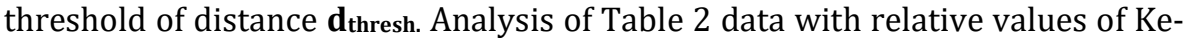
meny distance between rankings $\mathbf{d}_{\mathbf{i j}} \mathbf{i j}_{\mathbf{j}}$ shows that maximum extent of correspondence between experts' responses is slightly over $96 \%$. Thus, threshold value $\mathbf{d}_{\text {thresh. }}$ can amount to 0.05 or 0.1 , i.e. in this case, the responses with correspondence level of not less than 95 or 90 percent will be grouped. We shall accept dthresh. equaling to 0.1 .

Step 3. Let us convert matrix with relative values of Kemeny distance $\left\{\mathbf{d}_{\mathbf{i j}}\right\}$

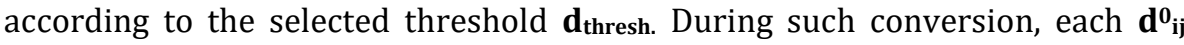

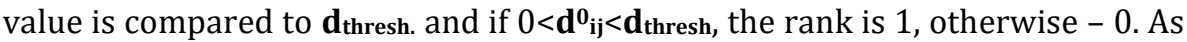
a result, we have Table 3 . 
Matrix of correlation between experts (responses)

Table 3 rankings at threshold $d_{\text {thresh }}=0.1$

\begin{tabular}{|l|l|l|l|l|l|l|l|l|l|}
\hline$\left\{\mathbf{d}_{\mathbf{i j}}\right\}$. & E1 & E2 & E3 & E4 & E5 & E6 & E7 & E8 & E9 \\
\hline E1 & $\mathbf{0}$ & $\mathbf{1}$ & $\mathbf{0}$ & $\mathbf{1}$ & $\mathbf{1}$ & $\mathbf{0}$ & $\mathbf{1}$ & $\mathbf{1}$ & $\mathbf{1}$ \\
\hline E2 & $\mathbf{1}$ & $\mathbf{0}$ & $\mathbf{0}$ & $\mathbf{1}$ & $\mathbf{1}$ & $\mathbf{0}$ & $\mathbf{1}$ & $\mathbf{1}$ & $\mathbf{1}$ \\
\hline E3 & $\mathbf{0}$ & $\mathbf{0}$ & $\mathbf{0}$ & $\mathbf{0}$ & $\mathbf{0}$ & $\mathbf{0}$ & $\mathbf{0}$ & $\mathbf{0}$ & $\mathbf{0}$ \\
\hline E4 & $\mathbf{1}$ & $\mathbf{1}$ & $\mathbf{0}$ & $\mathbf{0}$ & $\mathbf{1}$ & $\mathbf{0}$ & $\mathbf{1}$ & $\mathbf{1}$ & $\mathbf{1}$ \\
\hline E5 & $\mathbf{1}$ & $\mathbf{1}$ & $\mathbf{0}$ & $\mathbf{1}$ & $\mathbf{0}$ & $\mathbf{0}$ & $\mathbf{1}$ & $\mathbf{1}$ & $\mathbf{1}$ \\
\hline E6 & $\mathbf{0}$ & $\mathbf{0}$ & $\mathbf{0}$ & $\mathbf{0}$ & $\mathbf{0}$ & $\mathbf{0}$ & $\mathbf{0}$ & $\mathbf{0}$ & $\mathbf{0}$ \\
\hline E7 & $\mathbf{1}$ & $\mathbf{1}$ & $\mathbf{0}$ & $\mathbf{1}$ & $\mathbf{1}$ & $\mathbf{0}$ & $\mathbf{0}$ & $\mathbf{1}$ & $\mathbf{1}$ \\
\hline E8 & $\mathbf{1}$ & $\mathbf{1}$ & $\mathbf{0}$ & $\mathbf{1}$ & $\mathbf{1}$ & $\mathbf{0}$ & $\mathbf{1}$ & $\mathbf{0}$ & $\mathbf{1}$ \\
\hline E9 & $\mathbf{1}$ & $\mathbf{1}$ & $\mathbf{0}$ & $\mathbf{1}$ & $\mathbf{1}$ & $\mathbf{0}$ & $\mathbf{1}$ & $\mathbf{1}$ & $\mathbf{0}$ \\
\hline
\end{tabular}

It turns out that at $95 \%$ and $90 \%$ consistency levels a group of seven related experts' responses can easily be singled out: 1, 2, 4, 5, 7, 8, 9. Responses of experts 3 and 6 are not consistent with the rest.

Step 4. We search for the ranking that is the most consistent with the group of related responses singled out. Besides, consistent ranking must be a point that is the most corresponding to a set of possible sortings. But in case of a large number of experts, calculations can be simplified significantly if only responses of expert review participants are taken into account. If sampling is quite large, probability of getting consistent sorting in question is close to one. For that matter Table 1 is converted to Table 4 and $\boldsymbol{\Sigma} \mathbf{d}_{\mathbf{i j}}$ and $\boldsymbol{\Sigma} \mathbf{d}^{\mathbf{2}_{\mathrm{ij}}}$ values are calculated. Rank of an expert whose $\boldsymbol{\Sigma} \mathbf{d}^{2}{ }_{\text {ij. }}$. value is minimum, is deemed the most consistent with the group of responses singled out.

Table 4

Kemeny distances $d_{\mathrm{ij}}$ between rankings 1, 2, 4, 5, 7-9

\begin{tabular}{|c|c|c|c|c|c|c|c|c|c|}
\hline$\left\{d_{i j}\right\}$. & E1 & E2 & E4 & E5 & E7 & E8 & E9 & $\Sigma \mathbf{d}_{\mathrm{ij}}$ & $\Sigma \mathrm{d}^{2}{ }^{2}$ \\
\hline E1 & $\mathbf{0}$ & 2 & 4 & 4 & 4 & 2 & 2 & 18 & 60 \\
\hline E2 & 2 & $\mathbf{0}$ & 4 & 2 & 2 & 4 & 4 & 18 & 60 \\
\hline E3 & 28 & 42 & 24 & 36 & 42 & 36 & 24 & 232 & 8056 \\
\hline E4 & 4 & 4 & 0 & 4 & 2 & 2 & 2 & 18 & 60 \\
\hline E5 & 4 & 2 & 4 & 0 & 2 & 4 & 2 & 18 & 60 \\
\hline E6 & 18 & 35 & 48 & 48 & 28 & 42 & 32 & 251 & 9729 \\
\hline E7 & 4 & 2 & 2 & 2 & 0 & 2 & 4 & 16 & 48 \\
\hline E8 & 2 & 4 & 2 & 4 & 2 & 0 & 4 & 18 & 60 \\
\hline E9 & 2 & 4 & 2 & 2 & 4 & 4 & 0 & 18 & 60 \\
\hline
\end{tabular}

As the data from Table 4 suggest, the ranking of expert 7 is the most consistent with the singled out group of interrelated responses. Responses of experts 3 and 6 are inconsistent with those of singled out group. Figure 2 shows it quite clearly. 


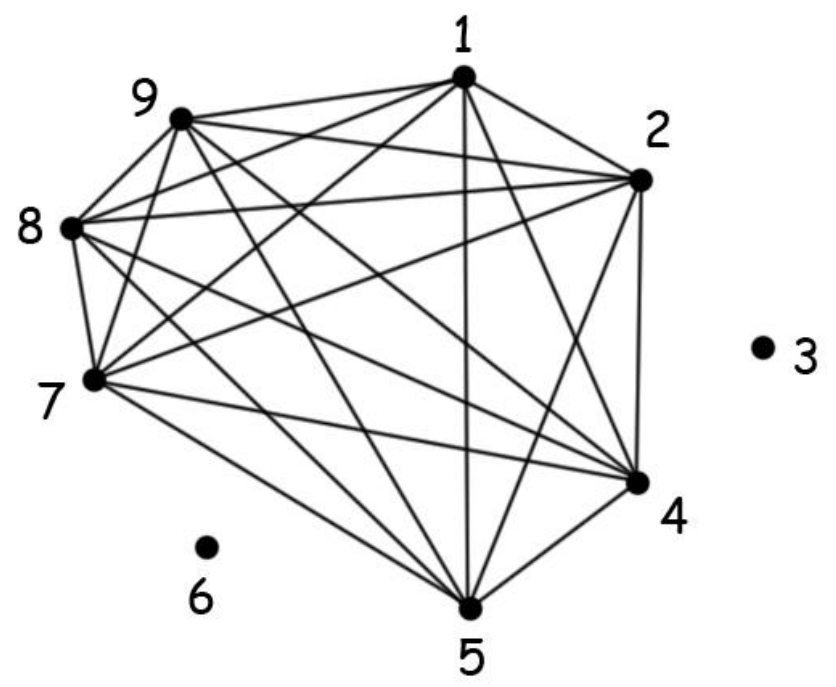

Figure 2. Graph of interrelations between rankings of experts from subgroup G1 (plotted based on Table 4)

\subsection{Assessment of Method Novelty and Perspective Utility: Method novelty: \\ 1) DELPHI method is used for ranking objects - factors, attributes, phe- nomena, etc. (not for futurism) \\ 2) For quantitative analysis of extent of repeatability of experts' opinions after each questionnaire, identification of matching expert groups and evaluation offeasibility of expert review completion, Kemeny distance method is used (meas- ure of proximity of linear order relation), while Kemeny median is used as a re- sulting ranking.}

3) For expert (participants of expert review) grouping who may have similar opinions regarding options of ranking of objects in question, threshold values of Kemeny distances between experts' reviews are set based on the probability of match of experts' opinions, e.g., close to $0.8 ; 0.9 ; 0.95 ; 0.99$.

4) Kemeny median is calculated in order to find an object ranking (sorting) the most consistent with group members in each of the expert groups created.

Method advantages: 1) Method soundness (soundness theorem was established in 1978 - Kemeny's median is the only resulting strict ranking that is neutral, consistent and condorcetian);

2) Increase of expert review result accuracy due to feedback from each questionnaire.

3) When using increment refinement of object rankings known advantages of DELPHI procedure are maintained (anonymity and possibility to get familiar with explanations supporting significantly differing answers), as are those of algorithm of search for consistent sorting of objects - accurate Kemeny's median and distance calculation. 
4) Timely use of both, DELPHI method, and suggested Kemeny's approach to object sorting made it possible:

*to obtain a meaningfully acceptable quantitative criterion for a reasoned completion of an expert review - a certain value (for example, $5 \%$ ) of the relative change in the total Kemeny distance is established.

*to form groups of experts, focusing on selected thresholds (depending on the degree of consistency of opinions) of Kemeny distance and to investigate the reasons for the relationship between expert assessments. Indeed, suppose that Figure 2 shows graphs of the relationship between the rankings of experts of extended subgroup G1 (including the developers of $\boldsymbol{n}$ competing projects). Moreover, in subgroup $\boldsymbol{A}$, in addition to the experts from subgroup G1 of the original composition, two project developers of $\boldsymbol{n}$ groups - identifiers 1 and 4 - were included. Graphs $\boldsymbol{B}$ and $\boldsymbol{C}$ have combined other developers of problem solutions. Now the causes for formation of such subgroups need to be analyzed.

*to find the most object sorting that is the most consistent with the members of each of expert groups by calculating precise or approximate Kemeny median,

5) Method of increment refinement of object rankings, while being straightforward and correct, does not have any limitations in number of expert review participants.

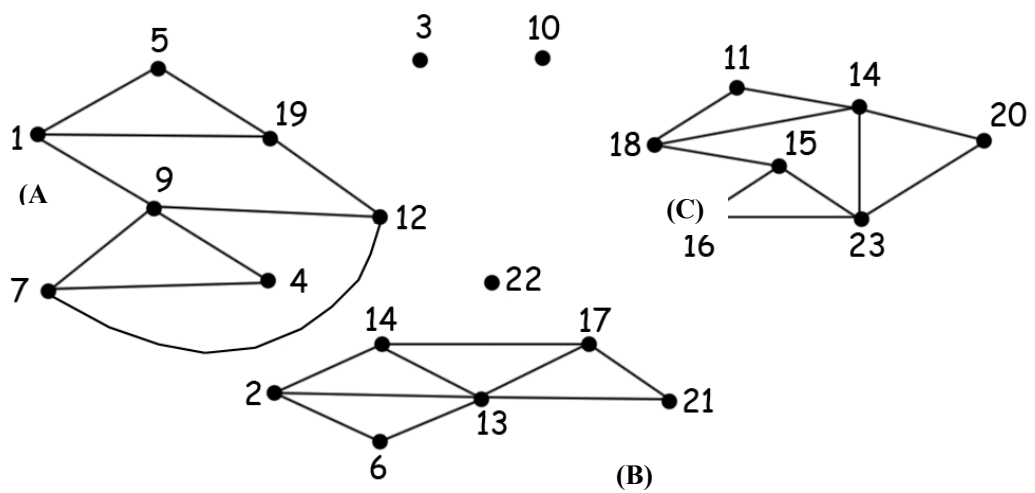

Figure 3. Graphs of relationship between the rankings of experts from subgroup G1 (extended composition)

Method trial and use: The method has been successfully tested *in solving different applied tasks; in scientific studies, incl. for object ranking in any area of expertise, to detect determinant factors, properties, attributes; in comparison and choice of design option, in comparison of software by the "Ease of Service and Maintenance" criterion, etc.

\subsection{Sequence of Steps of Forming Collective Choice (using PURO} method):

${ }^{*}$ Conversion of elements of Kemeny distance matrix obtained during the last step of expert questionnaire to relative units. Selecting threshold values for 
distance and assessment of response consistency extent within 80, 90 and 95 percent.

*Conversion of the matrix of relative values of Kemeny distance according to a threshold value selected.

${ }^{*}$ Grouping questionnaire respondents with the account of selected extent of consistency of expert opinions.

*Search for the ranking the most consistent with identified group of correlated responses using Kemeny's median.

3. Quantitative Assessment of Expenses and Efficiency (Task 3). For a comparative evaluation of the values of indicators characterizing the usefulness and effectiveness of the analyzed ways of solving the problem, it is proposed to use the method of step-by-step refinement of the values of the indicators with an estimate of the characteristics of the distribution of PUZ-OHR $[11,12]$.

3.1. Method Description. The method is, firstly, aimed at the use of a multistep procedure, at each step of which simulation modeling is performed, and, secondly, at integration of Delphi method with expert review aimed at getting generalized opinion of group of experts regarding potential range of values of an attribute in question.

Suppose that the integration with Delphi method is realized. But the question is how we can determine that collective mind has stabilized and further questionnaires can be stopped? For instance, what are the chances that a particular limit of an attribute in question will not be exceeded? What are the chances that the value of an attribute will be within the established confidence interval? List of such questions can be extended.

To answer the questions the following operations shall be implemented: Assessments of each $\mathbf{i}$ expert at step $\mathbf{j} \mathbf{E}^{\left(\mathbf{i}_{\mathbf{i}}\right.}$ are approximated by a rectangular (if an expert specified two values of an attribute) or triangular (when three values are specified) distribution (see [11-13]). Generalized collective opinion of $\mathbf{n}$ number of experts regarding value of an attribute in question is defined as a mean value of $\mathbf{n}$ number of random values with rectangular or triangular distribution by means of implementation at each step $\mathbf{k}$ of simulation modeling of function $\mathrm{E}^{(\mathbf{k})_{0 \sigma}}=\left(\boldsymbol{\Sigma} \mathrm{E}^{\left(k_{i}\right)}\right) / \boldsymbol{n},(\boldsymbol{i} \boldsymbol{E n})$. As a tool for implementation of simulation modeling software is used $[14,15]$, that enables creating a simulation model with minimum labor resources (in automatic mode). Simulation modeling at each step $\mathbf{k}$ results in statistical characteristics (mathematical expectation, variance, coefficient of variation, kurtosis, asymmetry) and distribution (table and histogram) of values of an attribute - function $\left.\mathbf{E}^{(k)_{0 \sigma}}=f\left(\mathbf{E}^{(k)}\right)_{i}\right)$. After each step (cycle of expert review) participants of expert group are made familiar with explanations supporting significantly differing assessments of attribute values, and offered change their previous responses if they wish. During each next step $\boldsymbol{j}$, change in values of coefficient of variance $\boldsymbol{K}^{\left(\boldsymbol{j}_{\mathbf{v}}\right.} \mathrm{var}_{\text {of function }} \mathbf{E}^{\left(\boldsymbol{( j}_{\mathbf{0}}\right.}$ is assessed. In case of deviation of coefficient of variance from previous value, e.g., by $\mathbf{5 \%}$ or less expert opinions may be deemed stabilized and expert review can be stopped. Based on the results of simulation modeling at the next step, confidential interval of attribute values is assessed as well as probability of its values being more or less than the established value. 
Automated synthesis of simulation models during implementation of the algorithm enables multifold reduction of labor resources needed for retrieval of attribute values in question.

3.2. Classification (Grouping) of Expert Review Participants. Using PUZ-OHR algorithm enables determination of the relationship between the responses of expert group members, extent of such relationship and factors influence of which explains presence of such relationship (whether or not education, place of work, job position, job experience, peculiarities of analyzed issue, etc. matter).

Suppose that after implementation of several steps of questionnaire for experts on the values of attributes in question, the responses of the respondents have stabilized at the last step (relative change in coefficient of variation has not exceeded the value established by the questionnaire organizers, for instance, 5 percent).

Moreover, the final table with experts' responses regarding the values of an attribute in question looks as the one shown in Table 5:

Table 5

Results of four (steps) cycles of expert review

\begin{tabular}{|c|c|c|c|c|c|c|c|c|c|c|c|c|}
\hline \multirow[b]{2}{*}{$\begin{array}{l}\text { Ex- } \\
\text { per } \\
t\end{array}$} & \multicolumn{3}{|c|}{ STEP 1} & \multicolumn{3}{|c|}{ STEP 2} & \multicolumn{3}{|c|}{ STEP 3} & \multicolumn{3}{|c|}{ STEP 4} \\
\hline & $\begin{array}{l}\mathbf{M i} \\
\mathbf{n}\end{array}$ & $\begin{array}{l}\text { Op } \\
t\end{array}$ & $\begin{array}{l}\text { Ma } \\
\mathbf{x}\end{array}$ & $\begin{array}{l}\text { Mi } \\
\mathbf{n}\end{array}$ & $\begin{array}{l}\text { Op } \\
t\end{array}$ & $\begin{array}{l}\text { Ma } \\
\mathbf{x}\end{array}$ & $\begin{array}{l}\text { Mi } \\
\mathbf{n}\end{array}$ & $\begin{array}{l}\text { Op } \\
t\end{array}$ & $\begin{array}{l}\text { Ma } \\
\mathbf{x}\end{array}$ & $\begin{array}{l}\mathbf{M i} \\
\mathbf{n}\end{array}$ & $\begin{array}{l}\text { Op } \\
\text { t }\end{array}$ & $\begin{array}{l}\mathbf{M a} \\
\mathbf{x}\end{array}$ \\
\hline$E_{1}$ & 3 & 5 & 9 & 5 & 6 & 9 & 6 & 7 & 9 & 6 & 7 & 9 \\
\hline $\mathrm{E}_{2}$ & 30 & 33 & 65 & 15 & 20 & 30 & 15 & 20 & 30 & 15 & 20 & 30 \\
\hline$E_{3}$ & 2 & 5 & 9 & 4 & 5 & 8 & 4 & 5 & 8 & 4 & 5 & 8 \\
\hline $\mathrm{E}_{4}$ & 8 & 11 & 15 & 8 & 11 & 15 & 8 & 11 & 15 & 8 & 11 & 15 \\
\hline $\mathrm{E}_{5}$ & 3 & 5 & 8 & 3 & 5 & 8 & 3 & 5 & 8 & 3 & 5 & 8 \\
\hline $\mathrm{E}_{6}$ & 14 & 17 & 22 & 14 & 17 & 22 & 14 & 17 & 22 & 12 & 14 & 20 \\
\hline $\mathrm{E}_{7}$ & 11 & 15 & 19 & 11 & 15 & 18 & 11 & 15 & 18 & 11 & 15 & 18 \\
\hline $\mathrm{E}_{8}$ & 20 & 25 & 55 & 15 & 20 & 35 & 10 & 12 & 22 & 10 & 12 & 21 \\
\hline $\mathrm{E}_{9}$ & 12 & 15 & 35 & 10 & 15 & 25 & 8 & 11 & 15 & 8 & 11 & 15 \\
\hline
\end{tabular}

*Bold font specifies values of an attribute changed by a respondent at the next step.

Now the task is to identify groups of experts whose opinions regarding the values of the attribute in question are quite similar, consistent.

Example of Expert Classification (Grouping). Table 6 shows the results of simulation modeling after each of four steps of expert review (see expert review results in Table 5).

[Note. Such a small number of experts given in the example is sufficient for explanation of specifics of grouping. It is obvious that on the Internet the number of subject matter experts involved in the expert review can amount to hundreds and thousands.] 
Results of modeling during steps 1-4

\begin{tabular}{|c|c|c|c|c|}
\hline Attribute & STEP 1 & STEP 2 & STEP 3 & STEP 4 \\
\hline Variable & $E^{(01)}$ & $E^{(02)}$ & $E^{(03)}$ & $E^{(04)}$ \\
\hline Number of iterations & 1000 & 1000 & 1000 & 1000 \\
\hline Mean & 17.51 & 13.7 & 12.24 & 11.98 \\
\hline Dispersion & 2.02 & 0.61 & 0.37 & 0.36 \\
\hline Standard deviation & 1.42 & 0.78 & 0.6 & 0.6 \\
\hline 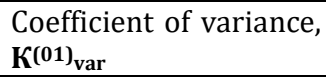 & 0.08 & 0.057 & 0.049 & 0.05 \\
\hline Asymmetry & 0.3 & 0.08 & 0.08 & 0.15 \\
\hline Kurtosis & -0.22 & -0.12 & -0.24 & -0.35 \\
\hline Minimum & 14.2 & 11.4 & 10.36 & 10.38 \\
\hline Maximum & 22.4 & 15.88 & 14.15 & 13.85 \\
\hline Modal interval & $\begin{array}{l}17.18: \\
17.93\end{array}$ & $\begin{array}{l}13.44: \\
13.84\end{array}$ & $\begin{array}{l}12.08: \\
12.43\end{array}$ & $\begin{array}{l}11.64: \\
11.95\end{array}$ \\
\hline
\end{tabular}

As Table 6 shows, expert review can be stopped since value $\mid \mathbf{K}^{(\mathbf{0 3})_{\text {var- }}}$

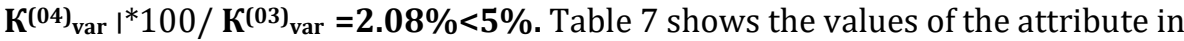
question obtained with probabilities of $0.8 ; 0.9$ and 0.95 as a result of simulation modeling of opinions of each of the experts.

Table 7

Value of attribute in question with probabilities of $0.8 ; 0.9 ; 0.95$

\begin{tabular}{|c|c|c|c|}
\hline \multirow{2}{*}{ Expert } & \multicolumn{3}{|c|}{ Probability } \\
\cline { 2 - 4 } & $\mathbf{0 . 8}$ & $\mathbf{0 . 9}$ & $\mathbf{0 . 9 5}$ \\
\cline { 2 - 4 } & \multicolumn{3}{|c|}{ Values of attribute in question } \\
\hline E1 & 7.9 & 8.2 & 8.55 \\
\hline E2 & 23 & 25.5 & 27.2 \\
\hline E3 & 6.5 & 6.9 & 7.3 \\
\hline E4 & 6.4 & 6.9 & 7.25 \\
\hline E5 & 6.25 & 6.75 & 7.2 \\
\hline E6 & 17 & 18 & 18.6 \\
\hline E7 & 15.8 & 16.3 & 17.1 \\
\hline E8 & 16.6 & 17.65 & 19.1 \\
\hline E9 & 12.55 & 13.4 & 14 \\
\hline
\end{tabular}

Results of grouping of experts according to whom values of attribute in question fell under the range with the probability that does not exceed $0.15 ; 0.3$; $0.5 ; 0.7 ; 0.85 ; 0.95$, per generalized (final) table of distribution, are given in Table 8. 
Related expert groups

\begin{tabular}{|c|c|c|c|c|c|}
\hline \multicolumn{6}{|c|}{ Probability } \\
\hline 0.15 & 0.3 & 0.5 & 0.7 & 0.85 & 0.95 \\
\hline \multicolumn{6}{|c|}{ Consistent value of attribute in question } \\
\hline 11.1 & 11.3 & 11.6 & 12 & 12.3 & 12.6 \\
\hline \multicolumn{6}{|c|}{ Related group of experts with selected probability of 0.8} \\
\hline $1,3,4,5$ & - & - & - & - & $\begin{array}{c}2,6,7,8 \\
9\end{array}$ \\
\hline \multicolumn{6}{|c|}{ Related groups of experts with selected probability of 0.9} \\
\hline $1,3,4,5$ & - & - & - & - & $\begin{array}{c}2,6,7,8 \\
9\end{array}$ \\
\hline \multicolumn{6}{|c|}{ Related groups of experts with selected probability of 0.95} \\
\hline $1,3,4,5$ & - & - & - & - & $\begin{array}{c}2,6,7,8 \\
9\end{array}$ \\
\hline
\end{tabular}

\subsection{Assessment of Method Novelty and Perspective Utility: Method}

Novelty. 1) Integration of Delphi procedure, usually used for futurism, with expert review aimed at obtaining value of an attribute in question;

2) Ensuring feedback during expert review through information subject matter experts about the results of the previous step;

3) Use of the program of successive steps, at each of which a complete cycle of expert review takes place;

4) Approximation of assessments of each of the experts represented by three values of an attribute in question (minimum, maximum and the most probable values) using a triangular distribution, and those represented by two values (minimum and maximum) - using a rectangular distribution;

5) Use of simulation modeling for determination of generalized collective opinion of $\boldsymbol{n}$ number of experts as a mean of $\boldsymbol{n}$ number of random values with a triangular or rectangular distribution (opinions of $\boldsymbol{n}$ number of expert group members);

6) Obtaining assessments of statistical characteristics (mathematical expectation, variance, coefficient of variation, kurtosis, asymmetry) and distribution of values of an attribute in question (in the form of a table and histogram);

7) Ability to determine that the values of attribute will not exceed a particular value or fall under the established range of values.

Method advantages: 1) Increased accuracy of results of expert review due to

${ }^{*}$ feedback from each next step;

*providing an expert with the possibility to specify three or two values for an attribute in question;

*determination of possibility for an attribute value to fall under the established range based on the results of simulation modeling.

2) Reduction of psychological overhead of an expert and negative impact of the presence of superiors and ambitious people on the results of expert review, since the anonymity is present and the experts do not communicate with 
one another and do not know who gave a particular justification supporting significantly differing values of an attribute.

3) Presentation of the total distribution as the mean (mathematical expectation) of the sum of the rectangular or triangular distributions of the estimates of individual experts makes it possible to obtain the resulting distribution of attribute values, even if the experts specify two or three values and there is a large dispersion of estimates.

4) Identification of spontaneous groupings of experts, whose estimates relative to the values of an attribute in question are close, makes it possible to investigate the reasons for the formation of such groups. Formation of expert groups is carried out based on a given threshold value of probability of a particular range of values of an attribute analyzed.

Method Use. The method is quite commonly used for both scientific studies and for education process. Economic feasibility has, therefore, been shown for the use of method for assessment of values of demand for a particular commodity, time of performance a particular business operation, losses of potential adverse sanctions of the competitors, time spent for breach if information system, estimated time for a problem solution, assessment of total cost of possessing logterm use items, for assessment of economic loss resulting from realization of security hazards of a company, etc.

3.4. Steps of Formation of Agreed Collective Choice (using PUZ-OHR algorithm):

*Implementation of simulation modeling of statements of each expert (approximation by triangular or rectangular distribution). Simulation modeling results in distribution of values of an attribute in question in the form of a histogram or a table for each expert;

*Values of an attribute are selected from the table that, for instance, will not be exceeded with the probabilities of $0.8 ; 0.9 ; 0.95$.

*Generalized distribution of attribute values is determined as a sum of opinions of all expert review members by simulation modeling.

*Table is generated listing number of experts whose opinions regarding attribute values in a generalized consistent distribution of values (from all experts) fell under the range with the selected probability.

4. Analysis of Results of Quantitative Assessment of Attributes and Realization of the Procedure of Choice (Task 4). To solve Task 4 PURO method and its modifications can be used. [16] describes one of its possible modifications used for solving Task 4.

CONCLUSION. Comparative advantages of the use of PURO and PUZOHR methods for formation of agreed collective choice during realization of expert reviews:

1) Activation of intellectual activity of expert due to providing the subject matter expert taking part in expert review with the possibility to consider objections and suggestions of other members of expert group in the atmosphere that is free of influence of personal qualities of the participants. Which is why there is no adverse influence of the presence of superiors and subordinates, friends and enemies, persons with different response speed, with different cultural and reli- 
gious backgrounds, etc. as expert group members on the results of the expert review since the experts: *do not, usually, know who participates in questionnaire; *do not know who gave what answer, who made what decision; *do not know who and how reasoned their decision, their positions; *and can be unaware of the true objectives of expert review survey organizers; *but they see at each step all justifications of the decisions of questionnaire participants and agreed opinion of experts

2) Formation of groups of experts, making collective choice, is done spontaneously, intuitively, without any outside pressure, without involvement of any external guiding subjects.

3) Possibility to use the so-called informed intuitive judgment of a subject matter experts by creating conditions for active, anonymous cooperation with other subject matter experts regarding different aspects of a problem in question. In this case, direct communication between subject matter experts is substituted with a sequence of steps, at each of which a complete cycle of expert review takes place, including informing subject matter experts about the results of the previous step.

4) On the Internet, the experts may be located in different cities and countries, and their number can be as much as hundreds and thousands. Therefore, among such subject matter experts there may be talented, naturally gifted individuals, who have competences in particular fields that are not related to the issue in question. And as practice suggests, these are the very people who, often times, become the originators of extraordinary suggestions, or provide the most persuasive justifications for choosing a particular project.

5) The use of the methods suggests correct quantitative determination of a moment (step number) for completion of an expert review survey (based on the value of change of the coefficient of variance or Kemeny distance).

6) The reliability of assessment results is increased by providing participants with the opportunity to substantiate and specify their answers; for example, to indicate the range of values of an attribute characterizing the project performance, and to obtain the statistical characteristics and distribution of the estimated attribute, as a result of subsequent simulation modeling and increment value refinement procedure.

7) The methods are correct, tested multiple times, and have proven their applied utility when used in different fields.

References:

[1] Khubaev Georgy. Assessment of the time required for the acquisition of knowledge //5th International Scientific Conference "Applied Sciences and technologies in the United States and Europe: common challenges and scientific findings" (New York, USA; February 12, 2014). Section 6. Pedagogy - New York, 2014. - p. 86-90.

[2] Khubaev Georgy. "On the Law of Distribution of Time for Acquisition of New Learning Material // 6th International Scientific Conference "System Analysis in Designing and Management" (SPb., June 28 - July 5, 2002). - SPb.: SPbGPU, 2002, - p. 431434. 
[3] Khubaev G. Management personnel classification by skill level and creativity // European science review. Section 14. Economics and management. - 2016. - No. 5-6. p. 223-228.

[4] Khubaev Georgy. Management personnel classification by skill level and creativity // Science and World. - 2016. - No. 5 (33). P.2. - p. 168-176.

[5] Khubaev Georgy. On one method of acquisition and formalization of apriori information when choosing significant factors // Collection of theses of Final Scientific Conference. Rostov Economical Institute. Issue 1. - Rostov-on-Don, 1973. - p. 238244.

[6] Khubaev Georgy. Machinery use effectiveness. - Rostov-on-Don: RSU, 1978. - 223 p.

[7] Olaf Helmer. Futurism: Delphi method. // Scientific and technical forecasting for industry and government agencies / transl. from English edited by G.M. Dobrov. - M.: "Progress", 1972. - p. 77-83.

[8] Helmer O. Social Technology, New York, Basic Books, Inc., Publishers, 1966.

[9] Kemeny J.G. Generalized random variables. Pacific Journal of Mathematics, vol. 9, 1959 , p. 1179-1189.

[10] Kemeny J., Snell J. Cybernetic modeling. Some applications. New York, 1963-1970. Transl. from English by B.G. Mirkina. edited by I.B. Gutchina M., 1972.

[11] Khubaev Georgy. Simulation modeling for retrieval of group expert opinion on the values of different attributes // AUTOMATION AND MODERN TECHNOLOGY. 2011. - No.11. - p. 19 - 23.

[12] Khubaev G. N. Stepwise determination of damage from realization of security hazards of a company //European Sciences review (Scientific journal). Section 13. Economics and management. - 2014. - No. 11-12. - p. 111-113.

[13] Khubaev G., Rodina O. Models, methods and software tools for assessment of total cost of possession of long-term-use items (on the example of software systems) / Monograph. - Saarbrucken: LAP LAMBERT Academic Publishing, 2012. - 370 p.

[14] System of automated synthesis of simulation models based on UML "SSM-UML" / Proprietors: Khubaev G.N., Shcherbakov S.M., Rvantsov Yu.A. // CeBIT 2015 (Hanover, 2015). Catalog of developments of the Russian Companies. Ministry of Education and Science of the Russian Federation; MSP ITT, 2015.

[15] Khubaev G.N., Shcherbakov S.M. System of automated synthesis of simulation models based on UML 2.0 (SSM-UML 2.0) // Certificate of state registration of software program. No. 2016661676. M.: Rospatent, 2016.

[16] Khubaev Georgy. Designing objects for different purposes: comparative assessment of appearance options // Sodruzhestvo (Russian and Chinese Scientific Journal). 2016. - No. 8. - p. 76-80. 